\title{
A Novel Fractional Sliding Mode Controller for Three-Phase Active Power Filter
}

\author{
Juntao Fei, Di Cao, and Yuncan Xue ${ }^{+}$ \\ College of IoT Engineering, Hohai University, Changzhou 213022, China
}

\begin{abstract}
This paper extends a conventional sliding mode controller with integer order to fractional ones for a three-phase active power filter (APF) in order to track instruction current quickly and precisely to offset the harmonic current in the electrical grid. A fractional sliding surface is utilized to make the system work on the designed sliding surface stably. The fractional sliding control system can obtain an extra degree of freedom apart from integer orders in order to improve the dynamic performance. Simulations are given to demonstrate the excellent performance of proposed controller. Comparisons with the conventional sliding mode controller show the smaller total harmonic distortion (THD) which is a significant index to evaluate the current quality in the smart grid.
\end{abstract}

Keywords: sliding mode control, three-phase active power filter, fractional control.

\section{Introduction}

Development of power technology has brought variety of benefits to our lives, however, accompanied by the dramatic changes, a lot of power quality problems have plagued us. For instances, due to the sinusoidal voltage applying non-linear load, fundamental current distort into harmonic current. Harmonic current will decrease the energy efficiency in production, transmission and utilization. Active power filter (APF) is a new power electronic device which can produce compensating currents into the grid in order to offset the harmonic currents that may do great harm to the electric power system. Since APF performs better than passive filter, APF possesses broad prospects in application and develops rapidly.

Because of the widely application of APF in power systems over the past few years, many scholars have devoted a large quantity of efforts to make APF work in a more efficient way by applying intelligent control methods and they have gotten great achievements. Wang et al. [1] presented comprehensive analysis and design for one-cycle controlled DC side APF on the basis of analyzing its circuit topology and basic principle. Rahmani et al. [2] derived a proportional-integral control law by linearizing the inherently nonlinear SAPF system so that the tasks of current dynamics and DC capacitor voltage dynamics became decoupled. Shu et al. [3] took a new approach using filed-programmable gate array (FPGA) to implement a fully digital control algorithm of active power filter. A common feature of the researches above is that they are based on the integer order. With the development of engineering applications, fractional calculus becomes widespread concerned these years. In the last three decades, many scientists applied fractional order controllers in order to achieve better performance and have also obtained some achievements. Wang et al. [4] addressed the design of sliding mode controller (SMC) for an uncertain chaotic fractional order economic system. By driving the state trajectory to the switching surface and maintaining a sliding-mode condition in spite of the uncertainties, Chen et al. [5] proposed the method for designing sliding-mode controller for a class of fractional-order linear interval systems with the external disturbances.

+ Corresponding author. Tel.: + 86-85192023.

E-mail address: johnfei123@163.com. 
The combination of intelligent controllers with fractional orders for APF can improve the tracking performance and stabilize the DC voltage in a short time. In this paper, a fractional fuzzy sliding mode control for three-phase active power filter is put forward. The conventional sliding mode controller is extended to fractional ones for three-phase active power filter. By applying fractional sliding mode controller to the system, the performance of APF is obviously better than that of the conventional control methods.By applying fractional modules to the sliding mode controller, the system can obtain an extra degree of freedom apart from integer orders. That means the fractional controller accesses more adjusted parameters while integer order controllers do not so that the performance of the controller can be improved.

\section{Mathematical Model of Active Power Filter}

The investigation in this paper is based on the shunt APF, especially parallel-voltage type of APF, since it is the most basic structure of APF. The block diagram of the three-phase three wire active power system is shown in Fig.1. The basic operating principle of active power filter is to detect the voltage and current produced by the operating system. After calculated by the current operation circuit, we can get the command signal $i_{c}^{*}$ of the compensating current. With the aim of achieving the compensating current $i_{c}$, the signal which is produced by the PWM need to be enlarged by amplifiers for its small amplitude. Only when compensating current offsets the harmonic current can we obtain the expected source current.

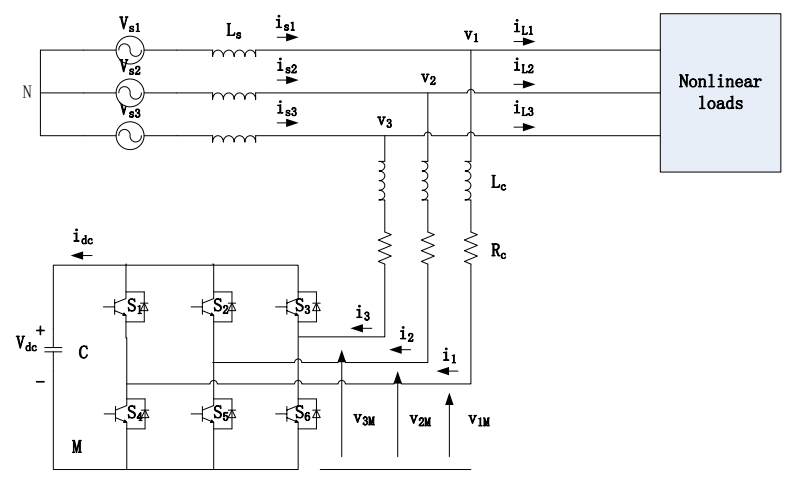

Fig. 1: Block diagram of main circuit of APF.

In the following section, the mathematical model of the APF will be given. According to the circuit structure of APF, we can obtain the following circuit equations by applying Kirchhoff rules to the circuitry.

$$
\left\{\begin{array}{l}
v_{1}=L_{c} \frac{d i_{1}}{d t}+R_{c} i_{1}+v_{1 M}+v_{M N} \\
v_{2}=L_{c} \frac{d i_{2}}{d t}+R_{c} i_{2}+v_{2 M}+v_{M N} \\
v_{3}=L_{c} \frac{d i_{3}}{d t}+R_{c} i_{3}+v_{3 M}+v_{M N}
\end{array}\right.
$$

In the equations, the parameter of $L_{c}$ and $R_{c}$ are the inductance and resistance of the APF respectively, $v_{M N}$ is the voltage between point $\mathrm{M}$ and $\mathrm{N}$.

It is assumed that the AC supply voltages are stable. Taking the three equations in (1) and combining with the absence of the zero-sequence in the three wire system currents yield the following equations:

$$
v_{M N}=-\frac{1}{3} \sum_{m=1}^{3} v_{m M}
$$

In order to indicate the working status of IGBT, we can define $c_{k}$ as the switch function which goes as:

$$
c_{k}=\left\{\begin{array}{l}
1, \text { if } S_{k} \text { is On and } S_{k+3} \text { is } \text { Off } \\
0, \text { if } S_{k} \text { is } \text { Off and } S_{k+3} \text { is } \text { On }
\end{array}\right.
$$

where $k=1,2,3$.

At the same time, taking $v_{k m}=c_{k} v_{d c}$ into consideration, thus (1) can be reformulated as 


$$
\left\{\begin{array}{l}
\frac{d i_{1}}{d t}=-\frac{R_{c}}{L_{c}} i_{1}+\frac{v_{1}}{L_{c}}-\frac{v_{d c}}{L_{c}}\left(\mathrm{c}_{1}-\frac{1}{3} \sum_{m=1}^{3} c_{m}\right) \\
\frac{d i_{2}}{d t}=-\frac{R_{c}}{L_{c}} i_{2}+\frac{v_{2}}{L_{c}}-\frac{v_{d c}}{L_{c}}\left(\mathrm{c}_{2}-\frac{1}{3} \sum_{m=1}^{3} c_{m}\right) \\
\frac{d i_{3}}{d t}=-\frac{R_{c}}{L_{c}} i_{3}+\frac{v_{3}}{L_{c}}-\frac{v_{d c}}{L_{c}}\left(\mathrm{c}_{3}-\frac{1}{3} \sum_{m=1}^{3} c_{m}\right)
\end{array}\right.
$$

The design of adaptive fractional fuzzy sliding mode control for three-phase active power filter is based on the above mathematical model (4).

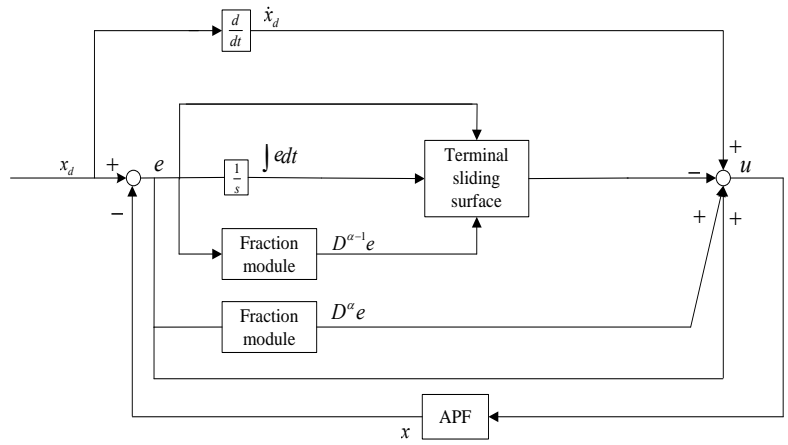

Fig. 2: Block diagram of fractional sliding mode control system for APF.

\section{Design and Analysis of Fractional Sliding Mode Controller}

Usually, calculus series are usually integer order, fractional calculus can extend the integration and differentiation to a more general form where its derivative and integral order can be a fraction.Define ${ }_{a} D_{t}^{\alpha}$ as the fundamental operator, where $a$ and $t$ are the bounds of the operation and $\alpha$ is the order of fractional calculus. For the purpose of simplifying the notation, in the following section, we denote the fractional derivative of order $\alpha$ as $D^{\alpha}$ instead of ${ }_{a} D_{t}^{\alpha}$. In this section, the design of fractional sliding mode controller will be given in detail, including how to make improvement on the controller and certify the stable performance of the system. The block diagram of fractional sliding mode control system for APF is shown in Fig.2.

The three equations in (4) can be transformed into the following form which is much more uncomplicated:

\[ \begin{array}{l}\dot{x}=f(x)+b u+d \\ \text { where } x=\left[i_{1}, i_{2}, i_{3}\right]^{T}, f(x)=-\frac{R_{c}}{L_{c}}\end{array} i_{k}+\frac{v_{k}}{L_{c}}, k=1,2,3 \text { and } b=-\frac{v_{d c}}{L_{c}} \text {, for the variance of } v_{d c}, b \text { can not be } \]
a definite value.

Assumption. $d$ is an unknown disturbance bounded such that $\rho-|d|>\sigma_{1}$.

where $\rho$ is a positive number. $\sigma_{1}$ is not only a positive number but also tiny enough.

The intention is that $x$ can track a given signal $x_{d}$. The tracking error is defined as $e=x_{d}-x$

Then the derivative of tracking error is: $\dot{e}=\dot{x}_{d}-\dot{x}$

Define a fractional order sliding surface as

$$
s=-\lambda_{1} e-\lambda_{2} \int e-\lambda_{3} D^{\alpha-1} e
$$

where $\lambda_{1}, \lambda_{2}, \lambda_{3}$ are designed and adjustable parameters. $\alpha-1$ is the fractional order of fractional derivate operation.

The derivative of the sliding surface is

$$
\dot{s}=-\lambda_{1} \dot{e}-\lambda_{2} e-\lambda_{3} D^{\alpha} e
$$

Substituting (10) into (12) yields

$$
\begin{aligned}
\dot{s} & =-\lambda_{1} \dot{e}-\lambda_{2} e-\lambda_{3} D^{\alpha} e \\
& =-\lambda_{1}\left(\dot{x}_{d}-f(x)-b u-d\right)-\lambda_{2} e-\lambda_{3} D^{\alpha} e
\end{aligned}
$$


The equivalent control force can be derived by setting $\dot{s}=0$ as

$$
u_{e q}=\frac{1}{b \lambda_{1}}\left[-\lambda_{1} f(x)-\lambda_{1} d+\lambda_{1} \dot{x}_{d}+\lambda_{2} e+\lambda_{3} D^{\alpha} e\right]
$$

Then, the sliding mode control signal is proposed as

$$
u=\frac{1}{b \lambda_{1}}\left[-\lambda_{1} f(x)-\lambda_{1} \rho \operatorname{sgn}(s)+\lambda_{1} \dot{x}_{d}+\lambda_{2} e+\lambda_{3} D^{\alpha} e\right]
$$

Choose a Lyapunov function as

$$
V=\frac{1}{2} s^{T} s
$$

Differentiate (11) with respect to time yields

$$
\begin{aligned}
\dot{V} & =s^{T}\left\{-\lambda_{1}\left[\dot{x}_{d}-f(x)-b \frac{1}{b \lambda_{1}}\left(-\lambda_{1} f(x)-\lambda_{1} \rho \operatorname{sgn}(s)+\lambda_{1} \dot{x}_{d}+\lambda_{2} e+\lambda_{3} D^{\alpha} e\right)-d\right]-\lambda_{2} e-\lambda_{3} D^{\alpha} e\right\} \\
& =s^{T} \lambda_{1}(d-\rho \operatorname{sgn}(s)) \leq\left|s^{T}\right| \lambda_{1}(|d|-\rho)
\end{aligned}
$$

According to the Assumption, $\rho-|d|>\sigma_{1}$ and $\lambda_{1}>0$, thus $\dot{V}<0$. This implies that $s(t)$ is bounded. Since $V(0)$ is bounded and $V(t)$ is bounded and non-increasing, we can conclude that $\int_{0}^{t}\left|s^{T}\right| \lambda_{1}(|d|-\rho) d \tau \leq V(0)-V(t) \leq \infty$. From (18), we can know $\dot{s}(t)$ is bounded. Correspondingly $s(t)$ is uniformly continuous. According to Barbalart lemma, $s(t)$ will asymptotically converge to zero, $\lim _{t \rightarrow \infty} s(t)=0$. It can be concluded that $e$ will asymptotically converge to zero, $\lim _{t \rightarrow \infty} e(t)=0$.

\section{Simulation Study}

In this section, a simulation example is presented to testify the proposed fractional sliding mode control and show the feasibility at the platform of Matlab/Simulink package with SimPower Toolbox. Comparison between controller with fractional modules and controller with integer orders is also given in this section to show the effectiveness of the proposed controller.

The main parameters of APF system are shown in Table I.

TABLE I: MAIN PARAMETERS

\begin{tabular}{l|l}
\hline \hline Supply voltage and frequency & $V_{s 1}=V_{s 2}=V_{s 3}=220 \mathrm{~V}, f=50 \mathrm{~Hz}$ \\
\hline Switching frequency & $f_{s w}=10 \mathrm{KHz}$ \\
\hline The non-linear load & $R=10 \Omega, L=2 \mathrm{mH}$ \\
\hline Active power filter parameters & $\begin{array}{l}L=10 \mathrm{mH}, C=100 \mu \mathrm{F} \\
v_{\text {dcref }}=1000 \mathrm{~V}\end{array}$ \\
\hline PI controller & $k_{p}=0.05, k_{i}=0.01$ \\
\hline \hline
\end{tabular}

In the fractional sliding mode controller, $\lambda_{1}=12, \lambda_{2}=3, \lambda_{3}=3$, we choose $\rho=180000$.

At the time $t=0.04 \mathrm{~s}$, the switch of compensation circuit is closed and then the APF starts to work. The source current of A phase before and after implementing fractional sliding mode control is shown in Fig. 3 and Fig.4. We can clearly see that the source current is equal to the load current before the APF is connected, however, the source current tends to a steady state after a half cycle which is about $0.01 \mathrm{~s}$. It is worth mentioning that the supply current is almost close to a sinusoidal wave which illustrating the good performance of APF in the steady state operation.

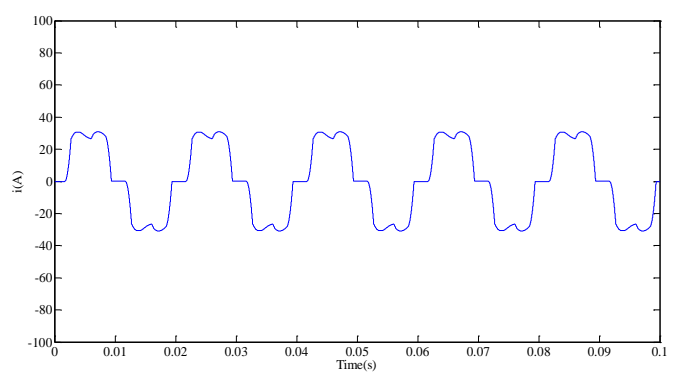

Fig. 3: The previous source current of A phase. 


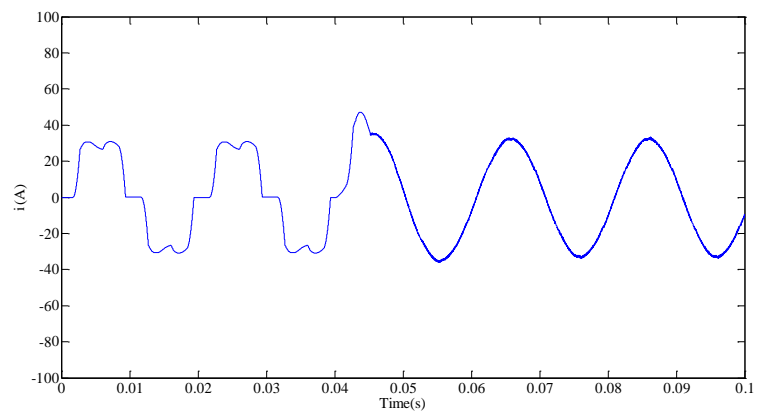

Fig. 4: The improved source current of A phase.

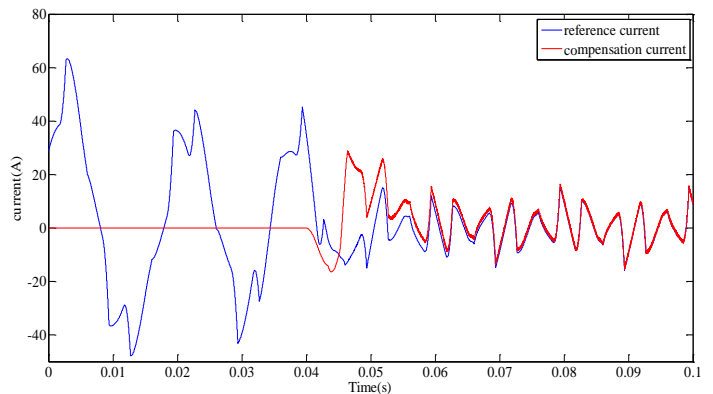

(b) $\alpha=0.6$

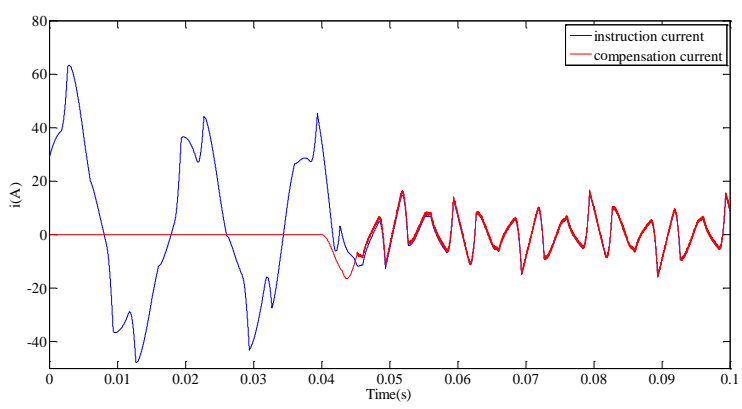

(c) $\alpha=0.9$

Fig. 5: Instruction current and compensation current with different values of fractional order.

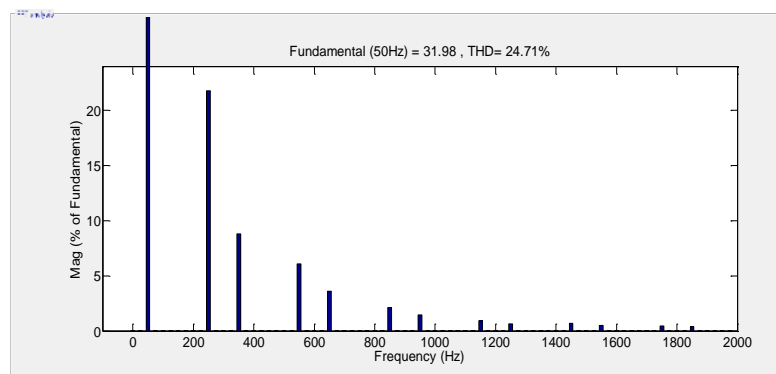

Fig. 6: Harmonic spectrum of source current at 0 s.

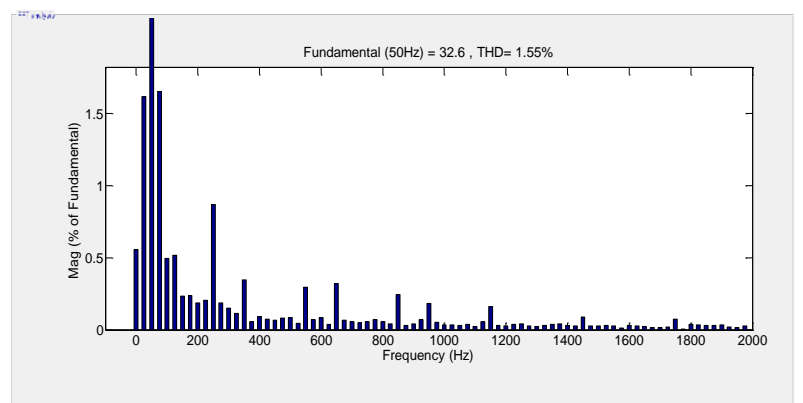

Fig. 7: Harmonic spectrum of source current at $0.06 \mathrm{~s}$ 


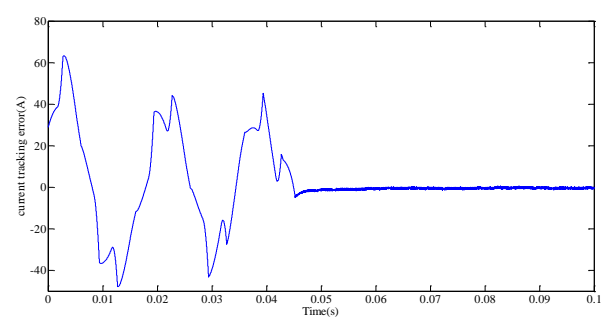

Fig. 8: Compensation current tracking error.

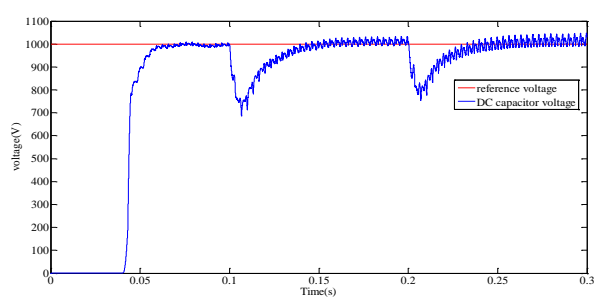

Fig. 9: DC capacitor voltage with the load increasing.

Instruction current and compensation current with different values of fractional order are given together in Fig.5. It can be seen that $\alpha=0.6$, the compensation current can gradually track the instruction current, however, the effect is not excellent and the time spent in tracking the current is too long. If we choose $\alpha=0.9$, it is obvious that the compensation current can quickly track the instruction current. So in the following simulation, we choose $\alpha=0.9$ in order to acquire better effects. Correspondingly, Fig. 8 shows the compensation current tracking error while $\alpha=0.9$, further verifying the effectiveness of the proposed fractional sliding mode control in tracking compensation current. Fig.6 shows the unqualified THD at the start of simulation. In Fig.7, after APF works, we can see that THD is $1.55 \%$, which is far less than the harmonic standard of IEEE of 5\%. It can be seen in Fig.9 that the DC capacitor voltage can also be adjusted to a stable status regardless of the changes of the applied load.

\section{Conclusion}

In this paper, a fractional sliding mode control for three-phase active power filter has been proposed and verified. First of all, a fractional sliding surface is proposed and the system is made to work on the designed siding surface stably. The fractional sliding mode controller is proved to be stable based on the Lyapunov analysis. The excellent dynamic performance, asymptotic stability and strong robustness are illustrated through the simulation results, and comparisons with the conventional sliding mode control show the small tracking error, good THD performance and fast stable DC voltage..

\section{Acknowledgment}

This work is partially supported by Natural Science Foundation of Jiangsu Province under Grant No. BK20171198. The Fundamental Research Funds for the Central Universities under Grant No. 2017B20014.

\section{References}

[1] Y. Wang, J. Li, Comprehensive analysis and design for one-cycle controlled DC side APF, proceedings of IEEE Conference on Industrial Technology, page: 750-755, 2006

[2] S. Rahmani, N. mendalek, Experimental design of a nonlinear control technique for three-phase shunt active power filter, IEEE Transactions on Industrial Electronics 57(10): page: 3364-3375, 2010

[3] Z. Shu, Y. Guo, Steady-state and dynamic study of active power filter with efficient FPGA-based control algorithm, IEEE Transactions on Industrial Electronics, 55(4), page: 1527-1536, 2008

[4] Z. Wang, X. Huang, Control of an uncertain fractional order economic system via adaptive sliding mode, Neurocomputing, 83, page: 83-88, 2012

[5] L. Chen, R. Wu, Adaptive sliding-mode control for fractional-order uncertain linear systems with nonlinear disturbances, Nonlinear Dynamics, 80(1-2), page: 51-58, 2015 